\title{
THE CONTRIBUTION OF ELECTRONICALLY EXCITED STATES TO THE RADIATION CHEMISTRY OF ORGANIC SYSTEMS
}

\author{
PROGRESS REPORT
}

SANFORD LIPSKY

Department of Chemistry

University of Minnesota

Minneapolis, MN 55455

June 30, 1988 - December 30, 1990

Prepared for the United States Department of Energy under Contract No.

DE-FG02-85ER13404

\section{DISCLAIMER}

This report was prepared as an account of work sponsored by an agency of the United States Government. Neither the United States Government nor any agency thereof, nor any of their employees, makes any warranty, express or implied, or assumes any legal liability or responsibility for the accuracy, completeness, or usefulness of any information, apparatus, product, or process disclosed, or represents that its use would not infringe privale ence herein to any specific commercial product, process, or service by its endorsement, recommanufacturer, or otherwise does not necessarily constitute or imply is mendation, or favoring by the United States Government or any agency the and opinions of authors expressed herein do not

United States Government or any agency thereof. 


\section{NOTICE}

This report was prepared as an account of work sponsored by the United States Government. Neither the United States Department of Energy, nor any of their employees, nor any of their contractors, subcontractors, or their employees, makes any warranty, expressed or implied, or assumes any legal liability or responsibility for the accuracy, completeness or usefulness of any information, apparatus, product or process disclosed or represents that its use would not infringe privately owned rights. 


\section{A. ABSTRACT}

The photocurrent from anthracene in 2,2,4-trimethylpentane, 2.2-dimethylbutane, cyclohexane, cyclopentane, and tetramethylsilane has been studied as a function of excitation energy from the ionization threshold to the onset of strong solvent absorption. In the case of 2,2,4-trimethylpentane (isooctane), the dependence of the photocurrent on the magnitude of an externally applied electric field was additionally studied in order to separately evaluate the effect of excitation energy on the electron ejection probability and on the geminate ion pair escape probability. The effect of excitation energy on the quenching by $n$-perfluorohexane of the anthracene photocurrent in isooctane is also reported.

The fluorescence from solutions of hexafluorobenzene in cyclopentane, 2,2,4-trimethylpentane, 2,2-dimethylbutane and tetramethylsilane irradiated with $\beta$-particles has been studied as a function of the hexafluorobenzene concentration from $c=$ $10^{-3}-10^{-1} \mathrm{M}$. The data are analyzed to permit extraction of the geminate ion-pair scavenging probability $\mathrm{p}^{\dagger}$. This is found to have a dependence on $c$ entirely similar to what has previously been reported for $\mathrm{p}^{\dagger}$ extracted from quenching of solvent fluorescence by perfluorocarbon scavengers, i.e., $p^{\dagger}=(\alpha c)^{0.7} /\left[1+(\alpha c)^{0.7}\right]$. The difference between these results and that for $\mathrm{p}^{\dagger}$ obtained via measurement of chemical product formation is discussed.

The absorption of $160 \mathrm{~nm}$ light by cyclohexane in mixtures of cyclohexane, benzene and tetraphenylmethylenediamine results in an emission spectrum consisting of the simultaneous fluorescence from all three components. A mechanism for the development of this spectrum and its dependence on benzene concentration is constructed and shown to be quantitatively consistent with the results of independent measurements on the separate components. In the absence of tetraphenylmethylenediamine, the quenching of cyclohexane fluorescence by benzene is found to be well represented by the standard diffusion model but with important contributions from "transient" terms. However, the concomitant 
sensitization of benzene fluorescence via energy transfer from cyclohexane is found to occur with an efficiency factor of only $0.26 \pm 0.02$ per encounter.

The fluorescence from both cyclohexane and benzene in their $\beta^{-}$irradiated mixtures has been studied as a function of benzene concentration from $\simeq 0.005 \mathrm{M}$ to $0.1 \mathrm{M}$. The quenching of the cyclohexane emission by benzene is found to be significantly greater than obtained under optical excitation conditions suggesting an important role for benzene as a scavenger of the geminate ion-pair precursor of the fluorescing state of cyclohexane. From diffusion models, a lower bound is established for the scavenging rate constant. An examination of the fluorescence from benzene leads to the conclusion that although the majority of benzene fluorescence derives from electronic energy transfer from excited cyclohexane, a large fraction (from $\simeq 20-50 \%$ over the concentration range studied) is generated by recombining molecular ions. The intrinsic efficiency with which the recombining ions generate fluorescent states of benzene, however, appears to be peculiarly low.

The fluorescence quantum yield of benzene vapor excited at $253.7 \mathrm{~nm}$ and at pressures from 10-50 torr has been determined at $22^{\circ} \mathrm{C}$ by comparison with the fluorescence from a dilute solution of benzene in cyclohexane. The value thus obtained is $0.044 \pm 0.006$ which is significantly lower than has been reported in all previous investigations. Although the origin of the discrepancy remains unknown, a theoretical argument is presented that tends to support the lower value.

The effect of benzene to quench the fluorescence of cis- and trans-decalin and to itself be sensitized to fluorescence has been studied for excitation of the system at $161 \mathrm{~nm}$. The dependence of the quenching of the decalin fluorescence on the benzene concentration (from 0.002 to $0.1 \mathrm{M}$ ) is shown to be fitted well for both isomers by the standard diffusion model. Corrections for static contributions are considered. The quenching encounter between excited decalin and benzene results in the production of an emitting state of benzene with an efficiency of only 0.44 for cis-decalin and 0.33 for trans-decalin. The 
origin of these low efficiencies appears to be attributable to inefficiency in the conversion to the emitting state of benzene from those states that are populated via the quenching encounter.

The fluorescence from $\beta^{-}$particle irradiation of cis- and trans-decalin containing benzene or toluene has been studied as a function of aromatic concentration from $\simeq 0.002 \mathrm{M}$ to $0.1 \mathrm{M}$ and over a spectral range that encompasses both the solvent and aromatic fluorescence. By comparisons with the fluorescence obtained using sub-ionization excitation of the decalin, the effect of benzene and toluene to intrude into the geminate ion-pair decay process has been extracted and rate constants for their scavenging action obtained via fitting to the standard diffusion model. The rate constants are compared to those reported from microwave conductivity studies on the "escaped" mobile hole in these liquids. For the reaction between trans-decalin ${ }^{+}+$toluene, the rates are in good agreement. However, for the reactions of either cis- or trans-decalin ${ }^{+}$with benzene, the rate constants extracted from the fluorescence analysis are about an order of magnitude larger. The discrepancies suggest the existence of differences in the internal energies and structures of the decalin positive ions when observed on the very short time scale of geminate recombination (probed in the fluorescence measurements) and that which is observed on the much loiger time scales that are probed in the microwave experiments.

An analysis of the development of aromatic fluorescence permits extraction of the fraction of aromatic fluorescence that derives from ionic recombination (as opposed to energy transfer) and the averaged efficiency of this recombination. In all of the systems studied here the ionic fraction remains high (i.e., $>20 \%$ ) even at millimolar concentrations of the aromatic. 


\section{B. RESEARCH}

\section{Photoconductivity of Anthracene in Liquid Hydrocarbons}

The photoconductivity of an aromatic solute in a liquid hydrocarbon exhibits a strong dependence on the nature of the hydrocarbon solvent. ${ }^{(1,2)}$ Both the energy threshold for photocurrent and the subsequent shape of the photocurrent spectrum are importantly altered by what would otherwise appear to be relatively minor changes in the nature of the solvent.

The effect of the solvent on the photocurrent threshold derives ultimately from its electronic polarization about the ion-pair. The cuntribution to the total polarization energy from the positive ion, although relatively large in magnitude, carries little solvent variability. On the other hand, the polarization energy deriving from the electron (which is essentially the negative of the "vertical" electron affinity of the solvent) appears to be very sensitive to molecular structure and accommodates almost all of the threshold shifting from one solvent to the next. ${ }^{(3)}$

The effect of the solvent on the structure of the photocurrent spectrum is less well understood. Holroyd, Preses, Bottcher, and Schmidt ${ }^{(2)}$ have noted that for anthracene in neopentane (and for azulene and methylnaphthalene as well) the photocurrent structure corresponds closely to the positions of relatively sharp Rydberg transitions in the vapor phase and have suggested that their manifestation in neopentane and their absence in n-pentane is ultimately tied to differences in the mobility of the electron in these solvents. The argument follows essentially that of Fermi's ${ }^{(4)}$ to explain the broadening of the spectral lines of high-lying Rydberg states of atoms by perturbers with large scattering amplitudes for slow electrons. By linking the electron's mobility in the solvent to the electron-solvent scattering amplitude, the argument for observation of the Rydberg transitions in neopentane and for their obscuration in n-pentane is made on the basis of a ca. 500 fold smaller electron mobility in $n$-pentane. To further support this, they note that with n-perfluorohexane added to anthracene (or benzanthracene) in neopentane, the 
photocurrent is quenched with an efficiency whose wavelength dependence exhibits a similar structure to that observed in the photocurrent spectrum. ${ }^{(2)}$ The implication is that the photocurrent quenching will be maximal when a Rydberg state of anthracene is populated. Such a state, it is presumed, will transfer an electron to $n$-perfluorohexane to produce a geminate ion-pair with a separation distance smaller (and, therefore, with smaller escape probability) than is produced at other excitation energies by the simple ejection of an electron and its subsequent "epithermal" scavenging by the perfluorocarbon. ${ }^{(1,2)}$

In the present study we reinvestigated the photocurrent spectrum of anthracene. We find that the Rydberg - state hypothesis is not a viable explanation to explain the structure in photocurrent spectra. Our measurements show that these structures reside not in the electron ejection probability but rather in the energy dependence of the thermalization range parameter of the electron. Considering the ejection as an autoionization from the valence states of the neutral that are initially populated by photon absorption, the structure is explainable in terms of final states of the process that leave the anthracene positive ion in some one of its vibrationally excited states. Solvent effects on the diffuseness of this structure can be qualitatively understood in terms of solvent modification of the autoionization amplitude either via alteration of the final state wavefunction of the quasi-free electron or via alteration of the Franck-Condon overlap of the valence and ion states due to differential shifts of the energies of these states. Also no evidence for structure in the photocurrent quenching efficiency by $n$-perfluorohexane for anthracene in isooctane was observed contrary to the reported appearance of such structure in neopentane solvent.

This work has been published (D. W. Tweeten and S. Lipsky, J. Phys. Chem. 1989, 93, 2683) and a reprint is attached. 
II. Dependence on Solute Concentration of the Efficiency of Scavenging of Electrons in Recombining Geminate Ion Pairs

When a simple hydrocarbon fluid is irradiated with fast electrons, an easily measurable fluorescence is observed which is identical to that produced by the absorption of non-ionizing photon radiation. The quenching of this fluorescence by added perfluorocarbons has been studied $(5)$ and found to depend on the fluorocarbon concentration, $c$, in such a way that the ratio, $Q_{e}$, of the yield in the absence, $G_{0}$, and in the presence, $G$, of the scavenger (i.e., $Q_{e}=G_{0} / G$ ) is given by

$$
Q_{e}=1+(\alpha c)^{0.7}
$$

where $\alpha$ is some empirical parameter.

If one assumes that i) the high-energy induced fluorescence derives exclusively from recombining geminate ion-pairs, ii) the fluorescence quenching is a consequence of capture of the "geminate" electron by the perfluorodecalin and its subsequent non-radiative recombination with the "geminate" positive ion and iii) electrons that are thus scavenged would otherwise (i.e., had they not been scavenged) have recombined with an "intact" solvent positive ion to yield a fluorescent solvent state, then it is simple to derive that

$$
Q_{e}=\left(1-p^{\dagger}\right)^{-1}
$$

where $\mathrm{p}^{\dagger}$ is the probability for scavenging of a geminate ion-pair (i.e., one that would have recombined in the absence of scavenger). ${ }^{(5)}$ By substituting eq. (1) into eq. (2), it then follows that the scavenging probability, $\mathrm{p}^{\dagger}$, is given by:

$$
\mathrm{p}^{\dagger}=\frac{(\alpha c)^{0.7}}{1+(\alpha c)^{0.7}}
$$

Eq. (3) appears, however, to be at variance with earlier studies of $\mathrm{p}^{\dagger}$ extracted from measurement of the yield of chemical product produced from the electron + scavenger attachment process over a similar range in c (i.e., $\simeq 10^{-4}$ to $10^{-1} \mathrm{M}$ ). From an analysis of a wide variety of such experiments, Warman, Asmus and Schuler ${ }^{(6)}$ found that $\mathrm{p}^{\dagger}$ could be 
represented by an equation similar to that of eq. (3) but with the power 0.7 replaced by 0.5 , i.e.,

$$
\mathrm{p}^{\dagger}=\frac{(\alpha c)^{0.5}}{1+(\alpha c)^{0.5}}
$$

Over the usual concentration range studied (of $\simeq 10^{-4}$ to $10^{-1} \mathrm{M}$ ), the difference between 0.7 and 0.5 would have been easily discernable.

To accommodate this disparity, Jonah and Sauer ${ }^{(7)}$ have recently suggested that not all geminate postive ion plus electron recombinations have the same probability for generating a solvent fluorescent state (i.e., assumption iii is violated). As a possible mechanism for this, they propose that solvent positive ions are initially generated in electronically excited states whose recombination with the electron gives neutral chemical products rather than fluorescence. Accordingly, only that fraction of the electron population which thermalizes sufficiently distant from the positive ion and whose recombination is therefore sufficiently slow to allow the positive ion to electronically relax, would give fluorescence. The consequence of this, they argue, is that whereas the technique for determining $\mathrm{p}^{\dagger}$ via measurement of the product formed from the scavenging reaction samples the entire population of electrons, the fluorescence quenching technique looks only at a smaller sub-population. From this they demonstrate that if the "total" scavenging probability is indeed given by eq. (4) then the $\mathrm{p}^{\dagger}$ extracted from the fluorescence quenching measurement must be of higher power than 0.5 in $c$, and, with not unreasonable choices of some pertinent parameters, could be as large as $0.7 .^{(7)}$

Since the chemical product technique is itself subject to the validity of some crucial approximations, we decided to utilize another technique for extracting $\mathrm{p}^{\dagger}$. In the case that recombination of the solvent positive ion and the solute negative ion generates a fluorescent state of the solute, it is possible, with some assumptions to extract another measure of $\mathrm{p}^{\dagger}$ from the concentration dependence of the intensity of this solute fluorescence. ${ }^{(8)}$ For this purpose, however, it is important that solute excited states derive predominately from the 
recombining ions and, therefore, that contributions from electronic energy transfer from neutral excited solvent states be kept negligible. To ensure this, we have employed three non-polar solvents from which no fluorescence has yet been observed (i.e., quantum yield less than $\left.10^{-6}\right),(9,10)$ namely 2,2-dimethylbutane (neohexane), 2,2,4-trimethylpentane (isooctane) and tetramethylsilane and one solvent, cyclopentane, which emits only a very weak fluorescence (quantum yield $\left.\simeq 5 \times 10^{-6}\right) .(10)$ These solvents were also chosen to span a large range in the mobility of the electron, and, therefore, a large range of rate constants for the electron attachment to the scavenger.

The choice of scavenger was dictated by three considerations. It should i) have a sufficiently large ionization potential to prevent transfer of an electron to the solvent positive ion (since if this were permitted to occur it would provide another channel for formation of solute excited states via recombination of scavenger positive and negative ions and thus complicate the experimental analysis), ii) have a sufficiently large electron affinity 80 as to favor the formation of the excited state of the scavenger over that of the solvent upon recombination of the scavenger negative and solvent positive ions, and iii) have an adequately large fluorescence quantum yield to permit its reliable observation above background levels. For these purposes, the scavenger of choice was hexafluorobenzene with an adiabatic ionization potential of $\simeq 9.9 \mathrm{eV},(11)$ an electron affinity of at least $0.5 \mathrm{eV}^{(12)}$ and a fluorescence quantum yield of $\simeq 0.034$.

In essence, what we find is that the $p^{\dagger}$ that we extract by the present technique continues to be well represented by eq. (3). contrary to the predictions of the Jon: $\mathrm{h}$ and Sauer model. Thus there remains a serious discrepancy tetween all of the fluorescence results and those obtained from chemical scavenging studies. Some possible origins of this discrepancy are considered in the paper that is based on this work. This paper has now been published (D. W. Tweeten, K. Lee and S. Lipsky, Radiat. Phys. Chem. 1989, 34, 771) and a reprint is attached. 
III. The Transfer of Electronic Energy from Cyclohexane to Benzene to Tetramethylphenylenediamine

We have recently reported on a peculiar effect of solution composition on the fluorescence intensity of a solute (T) dissolved in a mixture of cyclohexane (C) and benzene (B) when primary light absorption is by the cyclohexane. ${ }^{(14,15)}$ In these studies, the fluorescing state of $\mathrm{T}$ (i.e., $\mathrm{T}^{*}$ ) is generated indirectly via the two electronic energy transfer channels

$$
\mathrm{C}^{*}+\mathrm{T} \rightarrow \mathrm{C}+\mathrm{T}^{*}
$$

and

$$
\mathrm{B}^{*}+\mathrm{T} \rightarrow \mathrm{B}+\mathrm{T}^{*}
$$

where $\mathrm{C}^{*}$ and $\mathrm{B}^{*}$ are the fluorescing states of $\mathrm{C}$ and $\mathrm{B}$ and $\mathrm{B}^{*}$ is generated via

$$
\mathrm{C}^{*}+\mathrm{B} \rightarrow \mathrm{C}+\mathrm{B}^{*} \text {. }
$$

Since the channel II process should be more efficient than the channel I process (by virtue of the much longer lifetime of excited benzene), it was expected that the addition of small amounts of benzene to cyclohexane (at constant concentration of $T$ ) would cause an increase in the intensity of $\mathrm{T}^{*}$ fluorescence. Instead, a rather dramatic decline was observed.

To explain this, it was noted that if there were a fundamental inefficiency in the channel III process, then the addition of benzene, by subverting some $\mathrm{C}^{*}$ from channel I to channel III, would indeed lower the yield of $\mathrm{T}^{*}$ so long as the product of the efficiencies of channels II and III was less than the efficiency of channel I. To account for the channel III inefficiency, it was proposed that in the transfer of energy from $\mathrm{C}^{*}$ to $\mathrm{B}$, the propensity to preserve the total electronic energy of the system would cause there to be generated a large initial population of electronic states of benzene above $B^{*}$ (by virtue of the ca. $2 \mathrm{eV}$ disparity in electronic energies of $\mathrm{C}^{*}$ and $\mathrm{B}^{*}$ ) which would then internally convert to $\mathrm{B}^{*}$ with low probability. ${ }^{(16,17)}$ In the present investigation we have now quantitatively established this mechanism by simultaneous observations on the fluorescence of all three 
coniponents, cyclohexane, benzene and $\mathrm{T}$ as functions of benzene concentration when the cyclohexane is excited in its far UV absorption system at $160 \mathrm{~nm}$. Additional to this we have demonstrated that the quenching efficiency by benzene of cyclohexane fluorescence (excited at $160 \mathrm{~nm}$ ) can be explained adequately by a 1-state standard diffusion quenching model that includes "transient" effects. There appears to be no evidence for contributions to the quenching by interception by benzene of upper excited states of cyclohexane as has been previously suggested. ${ }^{(18)}$ Rather, the state initially populated at $\lambda_{e}=160 \mathrm{~nm}$ is shown to convert rapidly to the emitting state of cyclohexane, with an efficiency of 0.65 and that the quenching action of benzene is exclusively on this emitting state. In this quenching encounter, no electronic excitation appears to be lost, i.e., for every excited cyclohexane that is quenched, an excited benzene is generated, albeit not one that necessarily internally converts to the emitting state of benzene. Indeed, the probability for the internal conversion to the emitting state from the distribution of states initially populated by the energy transfer process is only 0.26 . Thus only about one in every four quenching encounters between excited cyclohexane and benzene results in the production of an emitting state of benzene.

A manuscript describing these results has been accepted for the Weller Issue of J. Phys. Chem. (D. B. Johnston and S. Lipsky, J. Phys. Chem., 1991). A preprint is attached.

\section{Energy Transfer From Cyclohexane to Benzene in Their Irradiated Mixtures}

The formation of excited solute states in irradiated saturated hydrocarbon liquids derives from both neutral and ionic channels. The neutral channel involves the formation first of an excited solvent molecule (either formed directly or via geminate electron + positive ion recombination) followed by non-radiative transfer of this excitation to the solute. The ionic channels are more complex involving capture by the solute (hereafter referred to as $\mathrm{B}$ ) of either the geminate electron, to form $\mathrm{B}^{-}$, or of the positive charge of the 
solvent (hereafter referred to as $\mathrm{C}$ ), to form $\mathrm{B}^{+}$, followed by some or all of the possible ionic recombination processes (i.e., $B^{+}+B^{-} ; B^{+}+e^{-}, B^{-}+C^{+}$).

The relative contributions of these various channels to the observed yields of excited solute states has been considered by many investigators, ${ }^{(19-24)}$ but remains generally unresolved. In only one case, that of biphenyl in cycloiexane, has there been some important progress made recently on this question using picosecond pulse radiolysis with analysis of both biphenyl negative ion and biphenyl fluorescence. ${ }^{(25)}$

In the case of benzene (or toluene or p-xylene) as solutes, the question of the mechanism of formation of excited aromatic takes on some additional relevance since the yields of these states (as deduced from their fluorescence) have been sometimes utilized to deduce $G$ values for excited solvent states $(19,26,27)$ under the assumption that all of the ionic channels are ignorable. Neither benzene nor toluene or $\mathrm{p}-\mathrm{xylene}$ are considered to form negative ions with any significant probability and their concentrations, in these studies, weee kept sufficiently low as to avoid, presumably, any appreciable contribution from gemir : te positive charge transfer processes. $(26,27)$ However, the $G$ values of excited solvent states thus obtained are found to be significantly lower than values determined by other methods. $(25,28,29,30)$ Although the question of possible origins for this disparity have been considered, $(27,29)$ there appears still no definitive answer.

By comparing the results obtained in our optical study of cyclohexane + benzene fluorescence (see section BIII of this Progress Report) with entirely similar experiments but using $0.22 \mathrm{MeV} \beta^{-}$particles as excitation source, we have been able to provide some new information pertinent to the mechanism of the formation of excited benzene.

The fraction of the total fluorescence from benzene that is attributable to electronic energy transfer from excited cyclohexane (formed by unperturbed $\mathrm{C}^{+}+\mathrm{e}^{-}$recomuinations) is computed quite generally from the data to vary from 0.83 to 0.47 over the benzene concentration range from 0.0056 to $0.112 \mathrm{M}$. The remainder of this fluorescence is due to recombining molecular ions which clearly make a non-trivial contribution until 
concentrations much less than $0.0056 \mathrm{M}$ are achieved. Accordingly, attempts to utilize benzene fluorescence to deduce the $\mathrm{G}$ value for excited cyclohexane by totally ignoring the ionic contributions, may be seriously in error.

A maruscript describing these results has been accepted for publication (D. B. Johnston and S. Lipsky, Rad. Phys. Chem. 1991, to be published). A preprint is attached.

\section{A Redetermination of the ${ }^{1} \underline{B}_{21} \longrightarrow A_{1 g}$ Fluorescence Quantum Yield of Benzene Vapor}

In 1965, Noyes, Mulac and Harter ${ }^{(31)}$ attempted a direct absolute measurement of the benzene vapor fluorescence quantum yield and obtained a value of $0.18 \pm 0.04$ at 253.7 $\mathrm{nm}$ over the pressure range from 8 to 14 torr. In 1977 another absolute value of $0.19 \pm 0.02$ at $254 \mathrm{~nm}$ was reported by Rockley ${ }^{(32)}$ using a photoacoustic technique Sinre then a variety of investigations ${ }^{(33-36)}$ into the fluorescence of single vibronic levels of benzene have utilized the $0.18-0.19$ value of vibrationally relaxed benzene as a reference quantum yield.

In the present investigation we have re-examined the benzene vapor fluorescence quantum yield. The impetus for this work came from some measurements we had begun in the Spring of 1990 on the effects of excitation energy on the emission spectrum and emission quantum yields of saturated hydrocarbon vapors. The emission quantum yields, when based on the $0.18-0.19$ benzene reference value, seemed to us to be unreasonably large. Accordingly, the decision was made to reinvestigate the benzene yield.

The technique that we have employed references the benzene vapor yield to that from a dilute solution of benzene in cyclohexane for which there is available a rather reliable value of $0.064 .^{(37-38)}$ The value that we have obtained for benzene vapor from $10-50$ torr and at $22^{\circ} \mathrm{C}$ is $0.044 \pm 0.006$, ca. $4 \times$ lower than the previously accepted standard value.

The discrepancy between our results and those of previous workers is, of course, disturbingly large, and we remain unsure as to the nature of its origin. Certainly the 
determination of a fluorescence quantum yield in one phase by comparison with the yield in another phase is fraught with a variety of potential sources of error and we make no claim to have eliminated them all. But we were totally unprepared for so large a discrepancy, the magi of which appears to indicate that, if the $0.18-0.19$ value is correct, then there must exist either a serious error in the solution quantum yield or a rather fundamental flaw in the comparison technique itself. The solution quantum yield appears to us to be essentially unassailable with substantial agreement amongst many laboratories ${ }^{(39)}$ hoth in its ratio to the standard (usually 9,10-diphenylinthracene) and also in the absolute quantum yield of the standard itself. $(37,40)$ On the other hand, with regard to the reliability of the $0.18-0.19$ value itself, we have ceveloped a theoretical argument that suggests that the vapor quantum yield could indeed be substantially lower. The argument is based on a formulation of the connection between the Einstein A and B coefficients that only appears to require for its validity the separability of molecular rotational and vibronic motions. ${ }^{(41)}$ Within this approximation, the calculation predicts a value for the fluorescence quantum yield for the thermally equilibrated population of the ${ }^{1} B_{2 u}$ state of 0.043 , surprisingly close to our experimental value.

A manuscript based on this work has been accepted for publication (D. B. Johnston and S. Lipsky, J. Phys. Chem., to be published). A preprint is attached to this report.

\section{The Sensitization of Benzene Fluorescence by Cis-and Trans-Decalin}

The quenching of cyclohexane fluorescence by benzene has been demonstrated ${ }^{(42)}$ to be well predicted by the standard Collins and Kimball diffusion model. $(43-45)$ However, observations on the concomitant appearance of benzene fluorescence indicates that in the quenching encounter, the annihilation of the cyclohexane emitting state (hereafter referred to as $\mathrm{C}^{*}$ ) is not completely compensated for by the creation of an emitting state of benzene (i.e., $\left.B^{*}\right)$. Conservation in number of emitting states appears to occur with a probability, $<\beta_{\mathrm{B}}>$, of only 0.26 per encounter. ${ }^{(42)}$ 
The factor of 0.26 is reasonably well predicted by assuming that there is, in fact, a conservation of total electronic energy in the $C^{*}+B$ encounters but that the upper electronic states of benzene that are thus populated do not convert with high efficiency to $\mathrm{B}^{*}$. ${ }^{(42)}$ The calculation utilizes Förster theory $(46)$ to deduce the population of upper excited states of benzene that are generated in the encounter (i.e., assumes that the population is determined from the overlap of the near-zone dipole fields associated with the solvent emittive and benzene absorptive radiative transitions) and utilizes previously determined experimental values of the internal conversion efficiencies from these states to $B^{*}$. (16)

In the present investigation we obtain encounter efficiencies of 0.33 and 0.44 for sensitization of benzene fluorescence by trans- and cis-decalin whose emitting states lie ca. $0.6-0.8 \mathrm{eV}$ below that of cyclohexane. These efficiencies, as expected, are larger than 0.26 but remain significantly below unity and are satisfactorily predicted using the aforementioned procedure which gives 0.32 for cis-decalin and 0.39 for trans-decalin. Certainly when these results are coupled with that obtained previously for benzene in cyclohexane (i.e., 0.26 experimentally and 0.28 predicted theoretically) and with some preliminary results on toluene in trans-decalin (i.e., 0.75 experimentally and 0.72 predicted theoretically) it would appear that our explanation for the encounter inefficiency in these systems is now essentially verified.

In this investigation we have also demonstrated that the standard diffusion model of Collins and Kimball ${ }^{(43)}$ continues to predict extremely well the efficiency with which benzene quenches the saturated hydroc arbon fluorescence in these much more viscous liquids.

A manuscript based on this work has been submitted for publication (D. B. Johnston, Y.-M. Wang and S. Lipsky, J. Phys. Chem., submitted) and a preprint is attached to this report. 
VII. Energy Transfer from Cis- and Trans-Decalin to Benzene in Their Irradiated

\section{Mixtures}

In a recent paper we reported on a comparison of the luminescence from cyclohexane + benzene mixtures when irradiated with fast electrons and with light absorbed by cyclohexane below its first ionization threshold. In this study, the fluorescence from both cyclohexane and from benzene were observed as functions of benzene concentration. From an analysis of the quenching effect of benzene on the cyclohexane fluorescence, it was possible to extract that fraction of the quenching attributable to an intrusion of the benzene on the cyclohexane ${ }^{+}+\mathrm{e}^{-}$geminate ion recombination. From this, it was concluded, that if the mechanism of intrusion involved either an electron attachment by benzene or involved positive charge transfer from cyclohexane ${ }^{+}$to benzene, then the rate constant for this "scavenging" action must be at least ca. $3.4 \times 10^{11} \mathrm{M}^{-1} \mathrm{sec}^{-1}$. Since this rate far exceeds reported upper bounds of ca. $10^{8}-10^{9} \mathrm{M}^{-1} \mathrm{sec}^{-1}$ for electron attachment by benzene, albeit in $n$-hexane, $(47,48)$ it appears plausible to conclude that either the positive ion is being scavenged or, alternatively, some non-scavenging mechanism is operating involving, for example, some non-radiative consequence of a temporary capture of the electron by benzene.

In order to assist in the resolution of this problem, it was decided to extend the previous investigation by replacing cyclohexane with cis- and trans-decalin. The purpose of this replacement being to examine the effect of benzene to intrude on the geminate recombination of $\mathrm{C}^{+}+\mathrm{e}^{-}$for two rather similar solvent positive ions, $\mathrm{C}^{+}$, but, for one of which, cis-decalin there had been reported a relatively low positive charge mobility of $2 \mathbf{x}$ $10^{-3} \mathrm{~cm}^{2} / \mathrm{sec}$ volt, as compared to $9 \times 10^{-3} \mathrm{~cm}^{2} / \mathrm{sec}$ volt for the trans-decalin. ${ }^{(49)}$ Additionally, for trans-decalin, it was decided to examine the effect of the replacement of benzene by toluene. This replacement was suggested by some very recent studies on positive ion scavenging in trans-decalin ${ }^{(50)}$ which provided rate constants for the 
disappearance of the microwave conductivity of $5 \times 10^{9} \mathrm{M}^{-1} \mathrm{sec}^{-1}$ for benzene and $2 \times 10^{11}$ $\mathrm{M}^{-1} \sec ^{-1}$ for toluene.

A final incentive for this study derived from results obtained in the cyclohexane + benzene study on analysis of the benzene component of the fluorescence which indicated a relatively inefficient production of excited benzene via ionic routes. The source of this inefficiency was not identified.. By changing the solvent system it was hoped to evaluate a possible contribution from instability of the geminate positive ion.

Our results indicate that the mechanism of benzene and toluene intrusion into the geminate recombination of decalin ${ }^{+}+\mathrm{e}^{-}$is due predominantly to the charge transfer from decalin ${ }^{+}$to the aromatic. Rate constants for this charge transfer have been extracted from the scavenging probability and are found to be about the same for benzene in cir- and trans-decalin $\left(k_{8} \simeq 8 \times 10^{10} \mathrm{M}^{-1} \mathrm{sec}^{-1}\right)$ but somewhat larger for toluene in trans-decalin ( $\mathbf{k}_{8}$ $\left.\simeq 2 \times 10^{11} \mathrm{M}^{-1} \mathrm{sec}^{-1}\right)$. The magnitude of these rate constants support the view of a fast bole mobility both in trans - and cis-decalins on the decalin ${ }^{+}+\mathrm{e}^{-}$-geminate recombination time scale. To explain the large disparity that has been reported in the mobilities of the "escaped" cis-and trans-decalin ions, we suggest that the cis-decalin positive ion must undergo some slow (ca. usec) structural modification that ultimately removes it from resonant interaction with the neutral solvent.

No evidence for positive ion instability has been found on the $\mathrm{C}^{+}+\mathrm{e}^{-}$geminate time scale. Indeed in both neat cis- and trans-decalins and in neat cyclohexane as well, an analysis of their $G$ values for production of emitting solvent states implies efficiencies for solvent ${ }^{+}+\mathrm{e}^{-}$recombination to give excited solvent states (which ultimately convert to the emitting states) which are close to unity.

Finally, analysis of the intensity of aromatic fluorescence in the cis- and trans-decalins indicate efficiencies for the ionic reactions that lead to this fluorescence to be larger than would be expected. A violation of the usual assumption, that energy 
transfer efficiencies to the aromatic are the same from $\mathrm{C}^{*}$ formed optically and that formed from $\mathrm{C}^{+}+\mathrm{e}^{-}$recombinations, is suggested as a possible contributor to this discrepancy.

A manuscript based on this work has been submitted for publication (D. B. Johnston, Y.-M. Wang and S. Lipsky, Radiat. Phys. Chem., submitted for publication). A preprint is attached to this report.

VIII. Ion-Electron Recombination Fluorescence

In 1981, Schwarz, Smith, Lias and Ansloos ${ }^{(51)}$ reported their results on the dependence on excitation energy of the fluorescence yield from some saturated hydrocarbon liquids. The excitation energy was varied from ca. 180 to $110 \mathrm{~nm}$ which spans a spectral region from the absorption onset of the first electronic transition to electron + positive ion continuum states that lie ca. 3-4 eV above the photoionization threshold. It was observed that, in general, the fluorescence intensity declined rapidly as the excitation energy, $E_{\text {exc }}$, increased, until the photoionization threshold was achieved (i.e., at $\mathbf{E}_{\text {exc }}=\mathrm{E}_{\mathrm{ion}}$ ) and from there on declined much more slowly. The initial rapid fall of of the intensity of fluorescence was a.tributed to fragmentation of the neutral excited states either to $B$ atom and radical hydrocarbon or to molecular hydrogen and olefin. The much slower decline in fluorescence intensity for $E_{\text {exc }}>E_{\text {ion }}$ was explained as due to stability of the cation on the time scale of geminate electron + positive ion recombination. Thus it was proposed that at $E_{\text {exc }}>E_{\text {ion }}$ an electron is ejected with increasing efficiency and kinetic energy as $E_{\text {exc }}-$ $E_{\text {ion }}$ increases. For those molecules which ionize [with efficiency $\varphi_{\text {ion }}\left(E_{\text {exc }}\right)$ ], the electron presumably always returns to an intact positive ion to generate a neutral state, at, or very close to the threshold state that photoejected. Those states that do not ionize, presumably continue to fragment and internally convert to the emitting state with low probability. Thus the intensity of fluorescence for a given $E_{\text {exc }},\left[I_{f}\left(E_{\text {exc }}\right)\right]$ should be proportional to $\left[1-\varphi_{\text {ion }}\left(\mathrm{E}_{\text {exc }}\right)\right] \beta\left(\mathrm{E}_{\text {exc }}\right)+\varphi_{\text {ion }}\left(\mathrm{E}_{\text {exc }}\right) \beta\left(\mathrm{E}_{\text {ion }}\right)$ where $\beta$ is the internal conversion efficiency to the emitting neutral solvent state. Clearly, by judicious choice of $\varphi_{\text {ion }}\left(E_{\text {exc }}\right)$, it would be 
possible to generate the observed behavior of $I_{f}\left(E_{\text {exc }}\right)$. Unfortunately, in the condensed phase, $\varphi_{\text {ion }}\left(E_{\text {exc }}\right)$ is not known reliably. Unlike the vapor phase, where ionization implies escape (and all charge can be collected), in the condensed phase only a small fraction of ionized states evolve into escaped pairs.

During the past few months, we have reexamined the dependence of $I_{f}\left(E_{\text {exc }}\right)$ on $E_{\text {exc }}$. Initially, this work was done to $150 \mathrm{~nm}\left(\mathrm{E}_{\text {exc }}=8.3 \mathrm{eV}\right)$ in order to provide us with data necessary for the interpretation of our energy transfer studies - ycoliminary extensions down to $140 \mathrm{~nm}\left(E_{\text {exc }}=8.8 \mathrm{eV}\right)$ seemed to indicate that $I_{f}\left(E_{\text {exc }}\right)$ might have a shallow minimum at $E_{\text {exc }} \simeq E_{\text {ion }}$. However, the exciting monochromators that were used in these measurements were not designed for the vacuum ultra-violet and excessive loss of light by reflections from too many optical surfaces reduced $\mathrm{I}_{\mathrm{f}}\left(\mathrm{E}_{\mathrm{exc}}\right)$ for $\lambda_{\text {exc }}<150 \mathrm{~nm}$ to levels so low as to make measurements unreliable. Accordingly, we have recently put together an improved system for this study. Preliminary results with cyclohexane clearly confirm the existence of a minimum in $I_{f}\left(E_{\text {exc }}\right)$ at $E_{\text {exc }} \simeq 8.8 \mathrm{eV}$ (ca. $\left.140 \mathrm{~nm}\right)$ followed by a subsequent slow rise to $\mathrm{E}_{\mathrm{exc}} \simeq 9.8 \mathrm{eV}$ of ca. $30 \%$.

The existence of the minimum implies that the ionization process is not, in some sense, reversible. Thus if $\mathrm{C}^{ \pm}$represents the threshold state for photoejection (i.e., $\mathrm{C}^{ \pm} \rightarrow$ $\left.\mathrm{C}^{+}+\mathrm{e}^{-}\right)$, then it 1aust follow that the recombination of $\mathrm{C}^{+}+\mathrm{e}^{-}$does not generate $\mathrm{C}^{ \pm}$but rather some lower slectronic state $\left(C^{* *}\right)$. In this way would be avoided the inefficiency of the $\mathrm{C}^{ \pm} \rightarrow \mathrm{C}^{* *}$ internal conversion and $\mathrm{I}_{\mathrm{f}}\left(\mathrm{E}_{\mathrm{exc}}\right)$ could increase.

There has been considerable recent theoretical interest in the rate of the $\mathrm{e}^{-}+\mathrm{C}^{+}$ recombination in dense fluids with particular concern for understanding the origin of rates which are less than predicted by the Debye equation. ${ }^{(52-55)}$ It has been suggested that in such cases, there is inefficiency in the loss of $\mathrm{e}^{-}$energy during the encounter of $\mathrm{C}^{+}+\mathrm{e}^{-}$. The results described in this investigation provide an experimental yandle on determining how much energy is lost and how such energy loss may be affected by temperature and nature of the scattering solvent. 


\section{Excited and Ionized States of Saturated Hydrocarbons}

The emission spectrum of cyclohexane liquid has been obtained with 2-photon excitation using the $308 \mathrm{~nm}$ output of a $\mathrm{XeCl}$ laser. The emission spectrum was examined as a function of delay time in order to seek evidence for a previously postulated long-lived metastable state of cyclohexane but, as yet, no conclusive evidence has been found. Within the past month we have attempted to probe for the metastable state by examining the temporal profile of the emission from toluene in cyclohexane. Thus far we have been experiencing stray light problems at the low toluene concentrations that are required in this investigation, and have, therefore, been, as yet, unsuccessful in confirming the predicted effect. This work is still, however, in progress.

Our research program with regard to study of the recombination kinetics of ionized states of saturated hydrocarbons has been seriously delayed by the lack of post-doctoral personnel. Two post-docs from the PRC who had accepted offers to come to Minnesota were ultimately unable to leave due to political problems. Finally, one of them, Dr. Yi-Ming Wang, did arrive in the early Fall of 1990. He is now setting up to work on the "Ionized State" problem but has already been most useful to us by assisting in our work on the energy transfer to benzene and toluene from cis- and trans-decalin (see Sections VI-VII). Also, we have been fortunate to successfully recruit Dr. Oleg Anisimov from the USSR to spend 10 months with us. He is scheduled to arrive in mid-January of 1991. Dr. Anisimov has had extensive experience in studying magnetic field effects on recombination fluorescence and will be of considerable help to us in this aspect of our program. Accordingly, we will be re-proposing this aspect of our program in our Renewal Proposal. 


\section{REFERENCES}

1. E. H. Böttcher, W. F. Schmidt, Proc. Tihany Radiat. Chem. 1982, 427.

2. R. A. Holroyd, J. M. Preses, E. H. Böttcher, W. F. Schmidt, J. Phys. Chem. 1984, 88, 744 .

3. R. A. Holroyd, J. M. Preses, N. J. Zevos, J. Chem. Phys. 1983, 79, 483.

4. E. Fermi, Nuovo Cimento 1934, 11, 157.

5. H. T. Choi, J. A. Haglund and S. Lipsky, J. Phys. Chem. 1983, 87, 1583.

6. J. M. Warman, K.-D. Asmus and R. H. Schuler, J. Phys. Chem. 1969, 73, 931; Ibid, Adv. Chem. Ser. 1968, No. 82, 25.

7. C. D. Jonah and M. C. Sauer, Jr., Rad. Phys. Chem., 1989, 34, 497.

8. F. P. Schwarz and D. Smith, Rad. Phys. Chem. 1985, 26, 257.

9. W. Rothman, F. Hirayama and S. Lipsky, J. Chem. Phys. 1973, 58, 1300.

10. H. Namba and S. Lipsky, J. Phys. Chem., unpublished data.

11. R. D. Levin and S. G. Lias, "Ionization Potential and Appearance Potential Measurements", NSRDS-NBS 71, 1982.

12. The electron affinity of $\mathrm{C}_{6} \mathrm{~F}_{6}$ in the gas phase as determined by electron transfer equilibria in $0.52 \mathrm{eV}$ : S. Chowdhury, E. P. Grimsrud, T. Heinis and P. Kebarle, J. Am. Chem. Soc. 1986, 108, 3630. A higher value of $1.09 \mathrm{eV}$ has been reported in non-polar liquids as determined by photodetachment: U. Sowada and R. A. Holroyd, J. Phys. Chem. 1980, 84, 1150.

13. Y. Beauchamp and G. Durocher, Spectrochimica Acta 1976, 32A, 269.

14. Y. Yoshida, L. Walter and S. Lipsky, Radiat. Phys. Chem. 1988, 32, 449.

15. Y. Yoshida and S. Lipsky, J. Phys. Chem. 1988, 92, 2240.

16. C. L. Braun, S. Kato and S. Lipsky, J. Chem. Phys. 1963, 39, 1645.

17. C. W. Lawson, F. Hirayama and S. Lipsky, J. Chem. Phys. 1969, 51, 1590.

18. K. Kimura and J. Hormes, J. Chem. Phys. 1983, 79, 2756.

19. G. A. Salmon, Radiat. Phys. Chem. 1976, 8, 13. 
20. F. Busi and L. Flamigni and G. Orlando, Radiat. Phys. Chem. 1979, 13, 165.

21. R. Hermann, O. Brede, J. Bös and R. Mehnert, Ber. Bunsenges. Phys. Chem. 1980, 84, 814.

22. S. Tagawa, Y. Katsumura and Y. Tabata, Radiat. Phys. Chem. 1982, 19, 125.

23. B. Brocklehurst, Int. Rev. Phys. Chem. 1985, 4, 279.

24. W. R. Appleton and B. Brocklehurst, Radiat. Phys. Chem. 1988, 32, 245.

25. Y. Yoshida, S. Tagawa, M. Washio, H. Kobayashi and Y. Tabata, Radiat. Phys. Chem. 1989, 4, 493.

26. J. H. Baxendale and J. Mayer, Chem. Phys. Lett. 1972, 17, 458.

27. F. Busi and G. Casalbore, Gaz. Chim. Ital. 1983, 113, 83.

28. L. Walter and S. Lipsky, Int. Radiat. Phys. Chem. 1975, 7, 175.

29. H. T. Choi, D. Askew and S. Lipsky, Radiat. Phys. Chem. 1982, 19, 313.

30. L. Wojnarovits and G. Földiak, Acta Chim. Acad. Sci. Hung. 1980, 105, 27.

31. W. A. Noyes, Jr., W. A. Mulac and D. A. Harter, J. Phys. Chem. 1966, 44, 2100.

32. M. G. Rockley, Chem. Phys. Lett. 1977, 50, 427.

33. C. S. Parmenter and M. W. Schuyler, Chem. Phys. Lett. 1970, 6, 339.

34. A. S. Abramson, K. G. Spears and S. A. Rice, J. Chem. Phys. 1972, 56, 2291.

35. W. R. Ware, A. M. Garcia, C. S. Parmenter, M. D. Schuh and K. Y. Tang, Chem. Phys. 1976, 17, 377.

36. M. Sumitani, D. O'Conner, Y. Takagi, H. Nakashima, K. Kamagawa, Y. Udagawa and K. Yoshihana, Chem. Phys. Lett. 1983, 97, 508.

37. I. B. Berlman, "Handbook of Fluorescence Spectra of Aromatic Molecules", 2nd Ed. Academic Press: New York, 1971, p. 108.

38. F. Hirayama and S. Lipsky, J. Chem. Phys. 1969, 51, 1939.

39. R. B. Cundall, D. A. Robinson and L. C. Pereira, Adv. Photochem. 1977, 10, 147.

40. S. Hamai and F. Hirayama, J. Phys. Chem. 1983, 87, 83.

41. T. A. Gregory and S. Lipsky, J. Chem. Phys. 1976, 65, 5469. 
42. D. B. Johnston and S. Lipsky, J. Phys. Chem. 1991, to be published.

43. F. C. Collins and C. E. Kimball, J. Colloid Sci. 1949, 4, 425.

44. A. Weller, Z. Phys. Chem. (Frankfurt am Main) 1957, 13, 355.

45. H. T. Choi and S. Lipsky, J. Phys. Chem. 1981, 85, 4089.

46. Th. Förster, Amm. Physik. 1948, $2,55$.

47. G. Bakale, E. Gregg and R. D. McCreary, J. Chem. Phys. 1972, 57, 4246.

48. J. H. Baxendale and E. T. Rasburn, Chem. Soc. Lond. Far. Trans. I, 1974, 705.

49. J. M. Warman, in "The Study of Fast Processes and Transient Species by Electron Pulse Radiolysis" (edited by J. H. Baxendale and F. Busi), Reidel, Dordrecht, 1982, p. 486 .

50. J. M. Warman, H. C. de Leng, M. P. deHaas and O. A. Anisimov, Radiat. Phys. Chem. 1990, 36, 185.

51. F. P. Schwarz, D. Smith, S. G. Lias and P. Ausloos, J. Chem. Phys. 1981, 75, 3800.

52. K. Shimsaka, M. Codama, Y. Nakamura, K. Serizawa and Y. Hatano, Radiat. Phys. Chem. 1989, 34, 519.

53. K. Shimsaka, M. Codama, T. Srithanratama, M. Yamamoto and Y. Hatano, J. Chem. Phys. 1988, 88, 7529.

54. M. Tachiya, J. Chem. Phys. 1987, 87, 4108.

55. M. G. Sceats, J. Chem. Phys. 1989, 90, 2666. 


\section{SUMMARY OF ATTACHMENTS}

1. D. W. Tweeten and S. Lipsky, "Photoconductivity of Anthracene in Liquid Hydrocarbons", J. Chem. Phys. 1989, 93, 2683. (reprint attached)

2. D. W. Tweeten, K. Lee and S. Lipsky, "Dependence on Solute Concentration of the Efficiency of Scavenging of Electrons in Recombining Geminate Ion-Pairs", Radiat. Phys. Chem. 1989, 34, 771. (reprint attached)

3. D. B. Johnston and S. Lipsky, "The Transfer of Electronic Energy from Cyclohexane to Benzene to Tetramethylphenylenediamine", J. Phys. Chem. 1991, Accepted for publication. (preprint attached)

4. D. B. Johnston and S. Lipsky, "Energy Transfer from Cyclohexane to Benzene in Their Irradiated Mixtures", Radiat. Phys. Chem., 1991, Accepted for publication. (preprint attached)

5. D. B. Johnston and S. Lipsky, "A Redetermination of the ${ }^{1} B_{2 u} \rightarrow{ }^{1} A_{1 g}$ Fluorescence Quantum Yield of Benzene Vapor, 1991, Accepted for publication. (preprint attached)

6. D. B. Johnston, Y.-M. Wang and S. Lipsky, "The Sensitization of Benzene Fluorescence by Cis- and Trans-Decalins", J. Phys. Chem., Submitted for publication. (preprint attached)

7. D. B. Johnston, Y.-M. Wang and S. Lipsky, "Energy Transfer From Cis- and Trans-Decalin to Benzene and Toluene in Their Irradiated Mixtures", Radiat. Phys. Chem., Submitted for publication. (preprint attached)

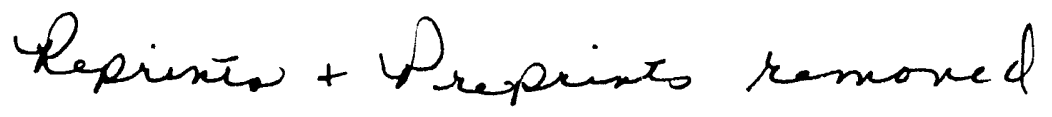


D. TIME DEVOTED TO PROPOSAL BY PRINCIPAL INVESTIGATOR

I. Academic Year $\quad 50 \%$

II. Summer $50 \%$

E. OTHER ACTIVITIES OF PRINCIPAL INVESTIGATOR

I. Teaching

Winter $1988 \quad$ Single quarter leave

Spring $1988 \quad$ General Chemistry (1004)

Fall $1988 \quad$ Chemical Thermodynamics (8512)

Winter $1989 \quad$ Mechanics and Reaction Kinetics (5535)

Spring 1989 General Chemistry (1004)

Fall 1989 Chemical Thermodynamics (5534)

Concepts of Chemistry (1014)

Winter $1990 \quad$ Honors Chemistry (1041)

Fall 1990 Introductory Quantum Mechanics \& Spectroscopy (8531)

Winter 1991 Introductory Quantum Mechanics \& Spectroscopy (8532)

Spring $1991 \quad$ General Chemistry (1005)

II. Extra-University Activities

1. Member of Evaluation Panel for Radiation Laboratory of University of Notre Dame 1988-1990.

2. Member of Evaluation Panel for Department of Chemistry, Argonne National Laboratory, 1990.

3. Guest Editor of Vol. 32 (Issues $1,2,3$ ) of the journal of Radiation Physics and Chemistry, 1988.

4. Chairman of Gordon Conference on Radiation Chemistry, 1988. 

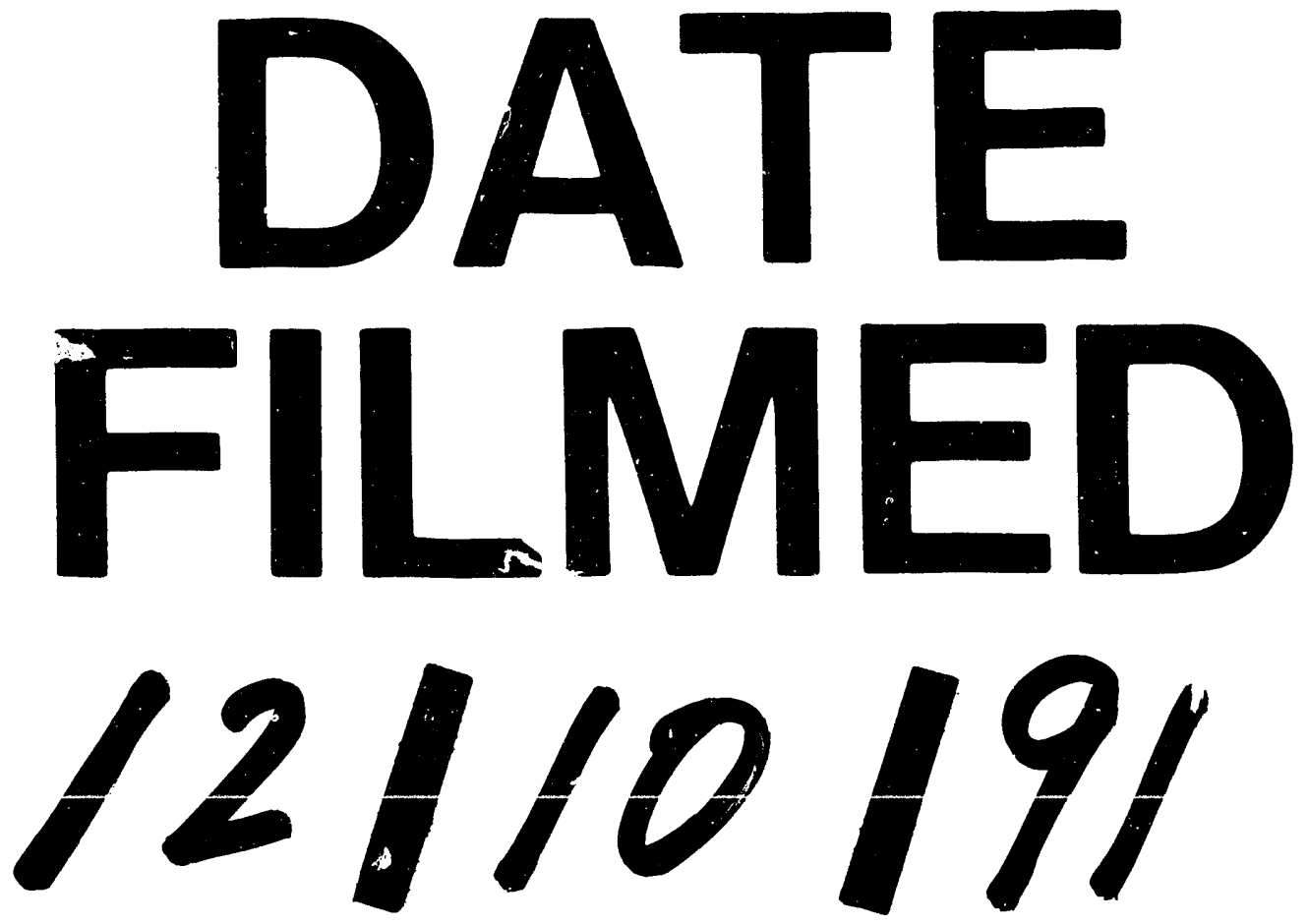
